\title{
Lymphocyte concanavalin A capping: a similarity between Down's syndrome and early onset primary degenerative dementia
}

\author{
M DUIJNDAM-VAN DEN BERGE, JG GOEKOOP \\ From the Psychiatric Hospital "Endegeest", Oegstgeest, The Netherlands
}

SUMMARY Lymphocyte capping with concanavalin A was studied in adult patients with Down's syndrome and aged patients with primary degenerative dementia. In both disorders a decreased capping was found as compared with age-matched and clinically relevant control groups. Colchicine had a strong enhancing effect on capping in Down's syndrome. In primary degenerative dementia the enhancing effect of colchicine was restricted to a subgroup of patients with onset of the dementing illness before the age of 80 years.

Epidemiological investigations by Heston $^{1}$ present evidence of an association between Alzheimer's disease and Down's syndrome. A microtubular disturbance has been proposed as the common underlying mechanism. Partial confirmation of this hypothesis has been established by Naeim et al $^{2}$ who demonstrated a decrease in concanavalin $\mathrm{A}$ induced capping of surface membrane receptors on lymphocytes of young adult patients with Down's syndrome. They also found an enhancing effect of colchicine, an agent which disturbs microtubule assembly, on concanavalin A capping in Down's syndrome.

We here present a study which replicates the experiments of Naeim et al in patients with Down's syndrome and extends the testing of the hypothesis of Heston with lymphocyte capping experiments in patients with primary degenerative dementia. Since we are specially interested in the clinical value of a reduced capping, the aged control groups consist of patients with multi-infarct dementia and patients with a functional psychiatric disorder.

\footnotetext{
Address for reprint requests: $M$ Duijndam-van den Berge, Psychiatric Hospital "Endegeest", Endegeesterstraatweg 5, 2342 AJ Oegstgeest, The Netherlands.
}

Received 22 December 1983 and in revised form 18 September 1985. Accepted 21 September 1985

\section{Subjects}

The study was performed in nine adult Down's syndrome patients (five males, four females; aged 33-57, mean $46 \pm 8$ years) and in 20 aged patients with primary degenerative dementia (10 males, 10 females; aged 48-86, mean $75 \pm 9$ years). Four patients had a presenile onset of the dementing illness, three of whom were younger than 65 years at the time of the study.

The control groups consisted of seven adult healthy staff members (three males, four females; aged 30-58, mean $42 \pm$ 9 years), 10 aged patients with multi-infarct dementia (eight males, two females; aged 66-92, mean $78 \pm 8$ years) and 29 aged patients with functional psychiatric disorder (five males, 24 females; aged 60-94, mean $75 \pm 8$ years). The diagnoses of dementia and functional psychiatric disorders were made according to DSM III criteria. ${ }^{3}$ Rosen's Ischemic Score $^{4}$ was used for differentiation of the dementias and Down's syndrome was verified for regular trisomy- 21 by karyotyping. The diagnoses in the functional psychiatric disorder group were schizophrenic disorder, paranoid and histrionic personality disorders, major depression and atypical psychosis. All patients were institutionalised and most had psychopharmacological medication (antidepressants, neuroleptics or benzodiazepines).

\section{Methods}

Lymphocytes were isolated from heparinised venous blood on a Histopaque-1077 gradient (Sigma, St Louis), washed twice in phosphate buffered saline (PBS, containing $120 \mathrm{mM} \mathrm{NaCl}, 8 \mathrm{mM} \mathrm{Na}_{2} \mathrm{HPO}_{4}, 1.5 \mathrm{mM} \mathrm{KH}_{2} \mathrm{PO}_{4}$ and $2.7 \mathrm{mM} \mathrm{KCl}, \mathrm{pH} \mathrm{7.4)}$ and resuspended in $1 \mathrm{ml}$ PBS. The cell 
suspension was divided into two test samples and two capping experiments per subject were run simultaneously, one with $10^{-4}$ colchicine, the other without colchicine. The cells were quickly cooled on ice and labelled with $100 \mu \mathrm{g} / \mathrm{ml}$ fluoresceinated concanavalin A (FITC-con A, Sigma) during 20 minutes at $4^{\circ} \mathrm{C}$. In the colchicine experiments colchicine was added 5 minutes prior to the addition of concanavalin A. After two quick washes with cold PBS $\left(4^{\circ} \mathrm{C}\right)$, containing $10^{-4} \mathrm{M}$ colchicine in the colchicine experiments, the cells were resuspended in $0.5 \mathrm{ml}$ of the corresponding washing medium and incubated at $37^{\circ} \mathrm{C}$ for 60 minutes. The cells were then fixed by adding $0.5 \mathrm{ml}$ of $4 \%$ paraformaldehyde for 15 minutes, washed twice in PBS and mounted in glycerol-PBS. The preparations were examined with a Leitz Orthoplan fluorescence microscope. Two hundred well labelled cells were counted per experiment and the rate of capping was expressed as the percentage of labelled cells with their fluorescence restricted to less than half of the cell circumference.

\section{Results}

The mean percentage of concanavalin A capped lymphocytes and the effects of colchicine are presented in table 1. In Down's syndrome a major reduction of concanavalin A capping was found as compared with the age-matched control group. The difference is significant at $p<0.001$, using the Mann-Whitney $U$ test, two-tailed. Compared with the adult controls all aged groups had a minor reduction of capping $(\mathrm{p}<$ 0.005 for each group). The percentage of capped cells was somewhat lower in primary degenerative dementia than in the other aged groups. This difference reaches statistical significance only in comparison with functional psychiatric disorder $(p=0.016)$.

The presence of colchicine did not influence the rate of capping in the adult controls and in the multiinfarct dementia patients. In the functional psychiatric disorder patients a slight enhancement was found. However, in Down's syndrome and primary degenerative dementia the presence of colchicine resulted in a strong enhancement of the rate of capping. The individual colchicine effects, expressed as the ratio of the percentage of capping in the presence of colchicine versus the percentage of capping without colchicine are shown in the figure. The figure suggests the existence of two primary degenerative dementia subgroups, one with an enhancing effect of colchicine, the other one without such an effect. A

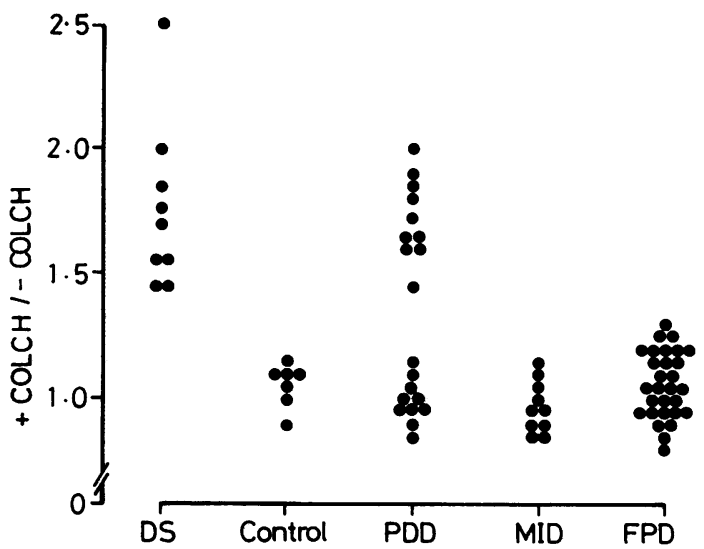

Fig The individual effects of colchicine on capping expressed as ratio of \% capped cells with colchicine $1 \%$ capped cells without colchicine.

The significance of the differences between the groups are calculated using the Mann-Whitney $U$ test, one-tailed.

$D S$ vs Adult controls: $p=0.0005, P D D$ vs FPD:

$p=0.018, P D D$ vs $M I D: p=0.0035$.

$D S=$ Down's syndrome.

$F P D=$ functional psychiatric disorder.

$P D D=$ primary degenerative dementia .

$M I D=$ multi-infarct dementia .

Table 1 Concanavalin A capping of lymphocytes and the effect of colchicine

\begin{tabular}{|c|c|c|c|c|}
\hline \multirow[t]{2}{*}{ Group (n) } & \multicolumn{2}{|l|}{$\%$ capped cells } & \multirow{2}{*}{$\begin{array}{l}\text { Significance of } \\
\text { colchicine } \\
\text { effect }\end{array}$} & \multirow[t]{2}{*}{ Ratio $\frac{+ \text { colchicine }}{- \text { colchicine }}$} \\
\hline & -colchicine & + colchicine & & \\
\hline $\begin{array}{l}\text { Adult Down's syndrome (9) } \\
\text { Adult contr. (7) } \\
\text { Aged primary degenerative dementia (20) } \\
\text { Aged multi-infarct dementia (10) } \\
\text { Aged functional psychiatric disorder (29) }\end{array}$ & $\begin{array}{l}14 \pm 4(9-18) \\
25 \pm 1(23-26) \\
17 \pm 4(9-24) \\
19 \pm 3(14-23) \\
20 \pm 3(13-27)\end{array}$ & $\begin{array}{l}24 \pm 3(19-27) \\
26 \pm 2(23-29) \\
22 \pm 3(17-29) \\
19 \pm 2(15-21) \\
21 \pm 4(15-29)\end{array}$ & $\begin{array}{l}\mathrm{p}<0.0005 \\
\mathrm{~ns}=0.002 \\
\mathrm{~ns}=0.01\end{array}$ & $\begin{array}{l}1.8 \pm 0.3(1.5-2.5) \\
1 \cdot 1 \pm 0.1(0.9-1 \cdot 1) \\
1.4 \pm 0.4(0.9-2 \cdot 0) \\
1.0 \pm 0.1(0.9-1 \cdot 1) \\
1 \cdot 1 \pm 0.1(0.8-1 \cdot 3)\end{array}$ \\
\hline
\end{tabular}

Results are given as mean \pm SD (range).

*Wilcoxon Signed-Ranks test, one tailed.

Table 2 The capping ratios in primary degenerative dementia patients in relation to the age of onset

\begin{tabular}{llllll}
\hline Age of onset $(y \boldsymbol{r})$ & $40-49$ & $50-59$ & $60-69$ & $70-79$ & $80-89$ \\
\hline Number of patients & 1 & 1 & 5 & 8 & 5 \\
Mean ratio & 1.60 & 1.63 & 1.69 & 1.31 & 1.00 \\
SD & - & - & 0.18 & 0.44 & 0.10 \\
\hline
\end{tabular}


strong negative correlation exists between the ratio and the degree of capping found in the experiments without colchicine. The Spearman correlation coefficient $r_{3}$ is -0.89 which is significant at $p<$ 0.001 . As a consequence in the primary degenerative dementia group high ratios correlate with low capping without colchicine.

As the four primary degenerative dementia patients with presenile onset of the dementing illness all had a high ratio, the relation between age of onset and the ratio was examined. A specification of the distribution of the ratios in separate categories with respect to age of onset is given in table 2 .

In the categories aged below 69 and beyond 80 years exclusively high respectively low ratios are present, as is shown by the small standard deviations of the mean ratio for these categories. In the category 70-79 years however both high and low ratios are present, resulting in an intermediate mean ratio with a high standard deviation. These data suggest that the ratios in primary degenerative dementia belong to two overlapping subgroups.

A Kruskal-Wallis one-way analysis of variance was performed in order to determine whether this distribution comes from one continuous population or not. For this purpose the three younger age categories were combined, thus reducing the test to $k=3$ samples. The calculated value of $\mathrm{H}=6.560$ is significant at $p<0.05$ implying that the data are not from one population.

In multi-infarct dementia and functional psychiatric disorder no correlation between age and capping ratio could be found. A Spearman Rank correlation test resulted in $r_{s}=-0.318$ and $r_{s}=-0.063$ respectively; both are not significant.

In order to estimate drug effects on the capping ratio the primary degenerative dementia group was split into the following six categories: no drug, antidepressants, neuroleptics and benzodiazepines only, and a combination of antidepressants and neuroleptics or benzodiazepines and neuroleptics. There was no relation between type of medication and ratio in primary degenerative dementia $(\mathrm{H}=3 \cdot 260, \mathrm{k}=6$, $\mathrm{p}=\mathbf{0} \cdot \mathbf{6}$, Kruskal-Wallis one-way analysis of variance). Preliminary studies with lumicolchicine showed no enhancement of the rate of capping in cases where colchicine did so.

\section{Discussion}

This study presents evidence of a similarity in lymphocyte concanavalin A capping between adult patients with Down's syndrome and aged patients with primary degenerative dementia. Compared with age-matched and clinically relevant control subjects a reduced concanavalin $\mathrm{A}$ capping and a strong enhancing effect of colchicine on capping is found in both conditions. This similarity is restricted to a subgroup of patients with primary degenerative dementia. Support for the existence of subgroups within primary degenerative dementia is based on the values of the capping ratios (a measure used for the individual colchicine effect) and the distribution of these ratios in different categories with respect to age of onset. The Kruskal-Wallis one-way analysis of variance shows that this distribution is not from one population. One subgroup is characterised by high ratios and onset before the age of 80 years, the other one by low ratios and onset after 70 years of age. Our findings confirm those of Naeim et al ${ }^{2}$ for Down's syndrome. Also the decreased capping we found in the aged control groups corresponds with the findings of these authors and others ${ }^{5}$ in aged subjects. In contrast with the findings of Naeim et $\mathrm{al}^{2}$ in healthy controls, we found no colchicine effect in multi-infarct dementia and only a slight effect in functional psychiatric disorder. This discrepancy concerning the colchicine effects can result from differences in our capping technique yielding higher percentages of capping $\left(60\right.$ minutes incubation at $37^{\circ} \mathrm{C}$ instead of 90 minutes on their experiments), but also from differences between the control groups in both studies. A more detailed comparison with their findings is not possible since they did not publish individual colchicine effects.

The similarity we found between Down's syndrome and a subgroup of primary degenerative dementia can be seen as a confirmation of the hypothesis of Heston ${ }^{1}$ which states that a similar disturbance of the microtubular system is involved in both illnesses. The fact that lumicolchicine, a colchicine derivative which does not affect the microtubular equilibrium, does not enhance capping indicates that microtubuli are involved indeed in the colchicine effects.

Our finding of a correlation between age of onset and abnormal microtubular functioning in primary degenerative dementia shows a parallel with the epidemiological data given by Heston ${ }^{6}$ of the risk for Down's syndrome among relatives of Alzheimer's disease probands. The high risk is extremely concentrated among relatives of probands with younger age of onset and declines to about that of the general population for relatives of probands with onset after 70 years of age. There is however a discrepancy between our data and the data given by Heston, ${ }^{6}$ since in our age of onset category of 70-79 years high ratios are still present. This may be explained by differences in estimating the age of onset or by the way the patients were selected. More research is needed to solve these problems. Our results imply that the capping ratio can be used as a marker of a specific subgroup of primary degenerative dementia characterised by a dis- 
order of the microtubular system. Further research on the aetiological role of a disturbance of this system and of pharmacotherapeutical consequences is warranted.

We thank Mrs S Koning and Mr W Dijksma for their assistance in the preparation of the manuscript.

\section{References}

${ }^{1}$ Heston LL. Alzheimer's disease, trisomy 21 and myeloproliferative disorders: associations suggesting a genetic diathesis. Science 1977;196:322-3.

${ }^{2}$ Naeim F, Bergmann K, Walford RL. Capping of con- canavalin A receptors on lymphocytes of aged individuals and patients with Down's syndrome: enhancing effect of colchicine; possible relation to microtubular system. Age Ageing 1981;4:5-8.

${ }^{3}$ Diagnostic and Statistical Manual of Mental Disorders. (Third Ed). The American Psychiatric Association, 1980.

${ }^{4}$ Rosen WG, Terry RD, Fuld PA, et al. Pathological verification of ischemic score in differentiation of dementias. Ann Neurol 1980;7:486-8.

${ }^{5}$ Noronha ABC, Antel JP, Roos RP, Arnason BGW. Changes in concanavalin A capping of human lymphocytes with age. Mech Ageing Dev 1981;12:331-7.

${ }^{6}$ Heston LL. Genetic studies of dementia: with emphasis on Parkinson's Disease and Alzheimer's neuropathology. In: Mortimer JA, Schuman LM, eds. The Epidemiology of Dementia. New York, Oxford: Oxford University Press, 1981:101-4. 